\title{
Selfing Can Facilitate Transitions between Pollination Syndromes
}

\author{
Carolyn A. Wessinger ${ }^{\star}$ and John K. Kelly \\ Department of Ecology and Evolutionary Biology, University of Kansas, Lawrence, Kansas 66045 \\ Submitted August 17, 2017; Accepted November 2, 2017; Electronically published March 14, 2018 \\ Online enhancements: appendixes. Dryad data: http://dx.doi.org/10.5061/dryad.8hc64.
}

\begin{abstract}
Aвstract: Pollinator-mediated selection on plants can favor transitions to a new pollinator depending on the relative abundances and efficiencies of pollinators present in the community. A frequently observed example is the transition from bee pollination to hummingbird pollination. We present a population genetic model that examines whether the ability to inbreed can influence evolutionary change in traits that underlie pollinator attraction. We find that a transition to a more efficient but less abundant pollinator is favored under a broadened set of ecological conditions if plants are capable of delayed selfing rather than obligately outcrossing. Delayed selfing allows plants carrying an allele that attracts the novel pollinator to reproduce even when this pollinator is rare, providing reproductive assurance. In addition, delayed selfing weakens the effects of Haldane's sieve by increasing the fixation probability for recessive alleles that confer adaptation to the new pollinator. Our model provides novel insight into the paradoxical abundance of recessive mutations in adaptation to hummingbird attraction. It further predicts that transitions to efficient but less abundant pollinators (such as hummingbirds in certain communities) should disproportionately occur in self-compatible lineages. Currently available mating system data sets are consistent with this prediction, and we suggest future areas of research that will enable a rigorous test of this theory.
\end{abstract}

Keywords: floral evolution, pollination syndrome, mating system, hummingbird pollination, Haldane's sieve, probability of fixation.

\section{Introduction}

Two common trends in the evolution of plant reproductive strategies are transitions in the primary pollinator and transitions from outcrossing to selfing (Stebbins 1970). In animal-pollinated species, floral traits are predicted to evolve in response to the most abundant and efficient pollinator in the community (Stebbins 1970; Waser et al. 1996), where efficiency is the rate of pollen transfer between visited flowers

\footnotetext{
* Corresponding author; e-mail: cwessinger@ku.edu. ORCIDs: Wessinger, http://orcid.org/0000-0003-3687-2559.
}

Am. Nat. 2018. Vol. 191, pp. 582-594. (C) 2018 by The University of Chicago. 0003-0147/2018/19105-57892\$15.00. All rights reserved. DOI: $10.1086 / 696856$
(Herrera 1987). When pollen is limiting, pollinator efficiency can determine fruit set per visit (Schemske and Horvitz 1984). Since pollinators differ in their receptiveness to floral signals and rewards as well as in how they interact with flowers, pollinator-mediated selection has led to the widespread convergent evolution of pollination syndromes - sets of floral traits associated with certain types of pollinators (Faegri and Van der Pijl 1979; Fenster et al. 2004; Harder and Johnson 2009). Pollinator communities vary over space and time, leading to repeated evolutionary transitions in pollination syndrome, as seen, for example, in transitions from bee to hummingbird syndrome (Grant 1994; Thomson and Wilson 2008; Abrahamczyk and Renner 2015).

A second important trend in plant evolution is the evolution of a self-compatible (SC) mating system from obligate outcrossing (Stebbins 1974; Igić et al. 2008). Selffertilization provides an inherent transmission advantage, all else being equal (Fisher 1941; Lloyd 1979, 1992), although this theoretical fitness gain can be countered by inbreeding depression, the fitness disadvantage of selfed offspring relative to outcrossed offspring (Lande and Schemske 1985; Harder and Wilson 1998). Therefore, selfing is more likely to be beneficial if it does not interfere with the production of outcrossed seeds (Lloyd 1992). For example, selfing may be favored as a mechanism to ensure reproduction if mates or pollinators are limited (Baker 1955; Eckert et al. 2006; Busch and Delph 2012). The benefits of selfing also depend on the mode and timing of self-fertilization (Lloyd 1979, 1992). Self-fertilization that occurs before or during the opportunity for outcrossing (prior or competing selfing) potentially expends or "discounts" gametes that otherwise could have generated outcrossed seeds. Selfing that occurs after the opportunity for outcrossing has passed (delayed selfing) does not usually carry this cost (Kalisz et al. 2004) and therefore may be broadly advantageous.

Historically, pollinator transitions and transitions to selfing have been considered separate evolutionary phenomena with independent underlying theories (Barrett 2002; Fenster et al. 2004; Busch and Delph 2012). Yet delayed selfing may 
benefit plant species with a specialized pollination system by offering reproductive assurance to counter the risk of pollination failure (Fenster and Martén-Rodriguez 2007). In support of this hypothesis, plant mating system studies posit an association between delayed selfing and specialization on a single type of pollinator, such as hummingbirds (Pérez et al. 2009; Martén-Rodríguez and Fenster 2010; Martén-Rodríguez et al. 2010). Theoretical work has examined the conditions that favor the evolution of pollinator specialization, including the influence of fluctuating pollinator abundances, local plant community structure, pollen competition, and trade-offs in pollinator attraction (Waser et al. 1996; Aigner 2001; Sargent and Otto 2005; Muchhala et al. 2010). Here we examine whether the potential to reproduce through selfing can facilitate evolutionary transitions between outcrossing mating systems that rely on different pollinators.

Mating system affects genetic transmission patterns within populations and can substantially impact the fate of new or rare beneficial mutations. In outcrossing populations, a new beneficial mutation is present only in heterozygotes. Its probability of surviving initial stochastic loss is approximately equal to $2 h s$, where $h$ is the dominance coefficient and $s$ is the selective coefficient (Haldane 1927). In such populations, dominant and partially dominant mutations should disproportionately contribute to adaptation, a theoretical prediction known as Haldane's sieve (Turner 1981; Orr 2010). By this logic, loss-of-function (LOF) mutations should contribute minimally to adaptation because they are often recessive $(2 h s=0$; Kacser and Burns 1981; Kondrashov and Koonin 2004; Phadnis and Fry 2005). Paradoxically, genetic studies have documented the contribution of LOF mutations to adaptation in a variety of organisms (reviewed in Hoekstra and Coyne 2007; Cutter and Jovelin 2015), suggesting that Haldane's sieve can be circumvented. One factor that can weaken the effects of Haldane's sieve is inbreeding, which acts to increase the frequency of homozygotes for a new recessive allele (Charlesworth 1992). At high selfing rates, the probability of fixation does not depend on dominance; instead, the dominance spectrum of new alleles that are fixed by selection is similar to the dominance spectrum for spontaneously arising beneficial mutations (Charlesworth 1992). In this way, an SC mating system can overcome Haldane's sieve. This prediction is supported by the observation that quantitative trait locus alleles underlying the evolution of domestication traits tend to be dominant or partially dominant in self-incompatible (SI) plants but not in SC plants (Ronfort and Glémin 2013).

Transitions to hummingbird pollination can be favored in some communities, since hummingbirds are predicted to be more efficient at transferring pollen than insect pollinators, such as bees (Castellanos et al. 2006). In contrast to hummingbirds, bees consume pollen, depleting the number of pollen grains they transfer between flowers. The prediction that hummingbirds are more efficient pollinators is supported by comparisons of pollen carryover by hummingbirds versus bees visiting Penstemon species (Castellanos et al. 2003). Transitions to hummingbird pollination are usually associated with the evolution of red, scentless flowers that are long and narrow and that produce large amounts of dilute nectar (Faegri and Van der Pijl 1979). The evolution of these traits often involves fixation of recessive alleles, many of which have been confirmed to be LOF mutations (i.e., in Mimulus cardinalis, Penstemon barbatus, and Petunia exserta; table 1). Despite being predominantly outcrossing, all three species are SC and are capable of autonomous selfing in the absence of pollinator visitation (Hiesey et al. 1971; Lange et al. 2000; Kokubun et al. 2006). We do not yet know whether facultative selfing in the absence of pollinator visitation (delayed selfing) facilitates floral evolution in response to pollinatormediated selection and/or alters the dominance spectrum of fixed beneficial mutations.

Here we test whether delayed selfing can facilitate a transition in pollination syndrome using a single locus population genetic model. Our work is motivated by the specific example of bees to hummingbirds but is general to any type of pollinator transition. In the model, the locus controls a trade-off in attractiveness to bees versus hummingbirds, which is consistent with previous observations of trade-offs in pollinator attraction. For example, flower color imposes a strong trade-off in attracting bees versus hummingbirds in reciprocal backcrosses between Mimulus lewisii (bee syndrome) and Mimulus cardinalis (hummingbird syndrome; Bradshaw and Schemske 2003). We find that delayed selfing expands the parameter conditions where hummingbird adaptation can evolve and increases the fixation probability for recessive alleles that underlie the evolution of traits to attract hummingbirds. A key prediction of our model is that lineages of plants that are SC should disproportionately evolve hummingbird pollination relative to obligately outcrossing lineages. Although we await comprehensive data sets that will enable a rigorous test of this prediction, an analysis of recent surveys of plant mating systems suggests that the prevalence of self-compatibility is higher in hummingbird-pollinated species than in their bee-pollinated relatives.

\section{Model and Results}

Consider a single locus where the ancestral genotype $A A$ is attractive to bees. A novel allele $a$ ("hummingbird allele") arises within this population that confers hummingbird attraction at the expense of bee attraction. We first find the conditions where the $a$ allele can invade a population fixed for the $A$ allele using a linear boundary analysis (see Otto and Day 2007). We then determine the fixation probability for the hummingbird allele when it arises as a single copy using a branching process that incorporates the stochas- 
Table 1: Genetic architecture of hummingbird attraction in three study systems

\begin{tabular}{|c|c|c|c|}
\hline Study system, adaptation & Gene action & Molecular basis & Reference(s) \\
\hline \multicolumn{4}{|l|}{$\begin{array}{l}\text { Mimulus cardinalis (hummingbird) } \times \\
\quad \text { Mimulus lewisii (bee): }\end{array}$} \\
\hline Gain of floral carotenoid production & Recessive & Unknown & $\begin{array}{l}\text { Bradshaw et al. 1998; Bradshaw } \\
\text { and Schemske } 2003\end{array}$ \\
\hline \multicolumn{4}{|l|}{ Increased floral anthocyanin } \\
\hline Loss of floral scent & Recessive & $\begin{array}{l}\text { LOF mutations to Ocimene synthase } \\
\text { and Limonene-myrcene synthase }\end{array}$ & Byers et al. 2014 \\
\hline \multicolumn{4}{|l|}{$\begin{array}{c}\text { Petunia exserta (hummingbird) } \times \\
\text { Petunia axillaris (moth): }\end{array}$} \\
\hline Red flower color & Recessive & Unknown & Hermann et al. 2013 \\
\hline Loss of UV absorption & Recessive & LOF mutation to $M Y B-F L$ & Sheehan et al. 2016 \\
\hline Loss of floral scent & Recessive & $\begin{array}{l}\text { LOF mutation to Cinnamate-CoA } \\
\text { ligase }\end{array}$ & Amrad et al. 2016 \\
\hline \multicolumn{4}{|l|}{$\begin{array}{c}\text { Penstemon barbatus (hummingbird) } \times \\
\text { Penstemon neomexicanus (bee): }\end{array}$} \\
\hline Red flower color & Recessive & $\begin{array}{l}\text { LOF mutation to Flavonoid } \\
3^{\prime}, 5^{\prime} \text {-hydroxylase }\end{array}$ & Wessinger and Rausher 2014 \\
\hline Large nectar volume & Recessive & Unknown & Wessinger et al. 2014 \\
\hline
\end{tabular}

Note: $\mathrm{LOF}=$ loss of function.

ticity of genetic segregation (Feller 1968; Pollak 1987). In our model, genotype controls pollinator attraction according to a symmetric trade-off (model parameters are listed in table 2). The magnitude of this trade-off is specified by an attraction coefficient, $\alpha$, that ranges from 0 to 1 . In appen$\operatorname{dix} \mathrm{A}$ (apps. A, B are available online), we relax the symmetry assumption. Attractiveness of heterozygotes to bees and hummingbirds is determined by $h$, the dominance coefficient. Genotype frequencies are given by $x_{0}(A A), x_{1}(A a)$, and $x_{2}(a a)$.
We assume that the plant species is hermaphroditic and, for simplicity, that each individual produces a single bisexual flower. A flower can be visited by a bee, visited by a hummingbird, or not visited. The probabilities of these three events depend on (1) the genotype-specific attractiveness to each pollinator, (2) the relative abundances of bees versus hummingbirds in the pollinator community, and (3) the overall degree of pollinator limitation in the community (table 3 ). The proportions of bees and hummingbirds in the pollinator community are given by $(1-Z)$ and $Z$, respectively; $Z$ can vary

Table 2: Description of model parameters

\begin{tabular}{ll}
\hline Parameter & \\
\hline$x_{0}$ & Frequency of $A A$ genotype \\
$x_{1}$ & Frequency of $A a$ genotype \\
$x_{2}$ & Frequency of $a a$ genotype \\
$\alpha$ & Attraction coefficient controlling the magnitude of the trade-off between \\
& bee and hummingbird pollination \\
$h$ & Dominance coefficient \\
$Z$ & Proportion of hummingbirds in the pollinator community \\
$1-Z$ & Proportion of bees in the pollinator community \\
$P$ & Degree of pollinator saturation \\
$E_{\mathrm{B}}$ & Efficiency of bee pollination \\
$E_{\mathrm{H}}$ & Efficiency of hummingbird pollination \\
$E_{\mathrm{DS}}$ & Efficiency of delayed selfing \\
$W_{\mathrm{I}}$ & Relative fitness of selfed progeny \\
$T_{\mathrm{B}}$ & Total frequency of bee visits to all genotypes \\
$T_{\mathrm{H}}$ & Total frequency of hummingbird visits to all genotypes \\
$m$ & Total contribution to the next generation (mean fitness) \\
$U_{1}$ & Fixation probability for the $a$ allele starting from a single $A a$ individual \\
$U_{2}$ & Fixation probability for the $a$ allele starting from a single $a a$ individual \\
\hline
\end{tabular}


Table 3: Genotype-specific attractiveness to each pollinator and per capita probabilities of visitation

\begin{tabular}{lccc}
\hline & $A A$ & $A a$ & $a a$ \\
\hline Attractiveness to bees & 1 & $1-h \alpha$ & $1-\alpha$ \\
Attractiveness to hummingbirds & $1-\alpha$ & $1-\alpha+h \alpha$ & 1 \\
Per capita probability of bee visitation & $V_{\mathrm{B} 0}=P(1-Z)$ & $V_{\mathrm{B} 1}=P(1-Z)(1-h \alpha)$ & $V_{\mathrm{B} 2}=P(1-Z)(1-\alpha)$ \\
Per capita probability of hummingbird visitation & $V_{\mathrm{H} 0}=P Z(1-\alpha)$ & $V_{\mathrm{H} 1}=P Z(1-\alpha+h \alpha)$ & $V_{\mathrm{H} 2}=P Z$ \\
$\begin{array}{l}\text { Per capita probability of not being visited by } \\
\quad\end{array}$ & $N_{0}=1-V_{\mathrm{B} 0}-V_{\mathrm{H} 0}$ & $N_{1}=1-V_{\mathrm{B} 1}-V_{\mathrm{H} 1}$ & $N_{2}=1-V_{\mathrm{B} 2}-V_{\mathrm{H} 2}$ \\
\hline
\end{tabular}

from 0 to 1 , but we explore the parameter space where $Z<$ 0.5 , since we do not expect a population to be ancestrally adapted to bees if hummingbirds are more abundant and more efficient at pollen transfer efficiency (see below). The size of the total pollinator community is represented by $P$ and varies from 0 to 1 (no pollination to saturating pollination). The opportunity for delayed selfing is inversely related to $P$.

The three different modes of pollination are associated with differences in seed set, which arise from differences in the relative efficiencies of pollen transfer (plants experience pollen limitation). We represent the relative efficiencies of bee-, hummingbird-, and delayed self-pollination as $E_{\mathrm{B}}, E_{\mathrm{H}}$, and $E_{\mathrm{DS}}$, respectively, and each can vary from 0 to 1 . Without loss of generality, we set $E_{\mathrm{H}}=1$, so that $E_{\mathrm{B}}$ and $E_{\mathrm{DS}}$ represent pollen transfer efficiencies relative to that provided by hummingbird pollination. Progeny of self-pollination experience a fitness deficit due to inbreeding depression; $W_{\mathrm{I}}$ denotes the viability of selfed progeny relative to outcrossed progeny. The product $E_{\mathrm{DS}} \cdot W_{\mathrm{I}}$ is thus the realized gain from self-pollination. When $E_{\mathrm{DS}}=0$, the plant population is SI. The relative abundances of each pollinator in the community as well as their pollen transfer efficiencies are treated as constant in the model.

Each individual in the population functions as a (potential) maternal plant and the probabilities of each paternal genotype depend on the mode of pollination. We determine the genotype frequencies in the next generation from Mendelian ratios of offspring, given the maternal and paternal genotypes (app. A). Genotype frequencies in the next generation $\left(x_{0}^{\prime}, x_{1}^{\prime}\right.$, and $\left.x_{2}^{\prime}\right)$ are calculated by adding together the contributions from each mode of pollination to offspring genotypes:

$$
\begin{aligned}
x_{0}^{\prime}=\frac{1}{m}[ & \frac{E_{\mathrm{B}}}{T_{\mathrm{B}}}\left(\left(x_{0} V_{\mathrm{B} 0}\right)^{2}+x_{0} V_{\mathrm{B} 0} x_{1} V_{\mathrm{B} 1}+\frac{1}{4}\left(x_{1} V_{\mathrm{B} 1}\right)^{2}\right) \\
& +\frac{E_{\mathrm{H}}}{T_{\mathrm{H}}}\left(\left(x_{0} V_{\mathrm{H} 0}\right)^{2}+x_{0} V_{\mathrm{H} 0} x_{1} V_{\mathrm{H} 1}+\frac{1}{4}\left(x_{1} V_{\mathrm{H} 1}\right)^{2}\right) \\
& \left.+E_{\mathrm{DS}} W_{\mathrm{I}}\left(x_{0} N_{0}+\frac{1}{4} x_{1} N_{1}\right)\right],
\end{aligned}
$$

$$
\begin{aligned}
x_{1}^{\prime}=\frac{1}{m}[ & \frac{E_{\mathrm{B}}}{T_{\mathrm{B}}}\left(x_{0} V_{\mathrm{B} 0} x_{1} V_{\mathrm{B} 1}+\frac{1}{2}\left(x_{1} V_{\mathrm{B} 1}\right)^{2}+2 x_{0} V_{\mathrm{B} 0} x_{2} V_{\mathrm{B} 2}+x_{1} V_{\mathrm{B} 1} x_{2} V_{\mathrm{B} 2}\right) \\
& +\frac{E_{\mathrm{H}}}{T_{\mathrm{H}}}\left(x_{0} V_{\mathrm{H} 0} x_{1} V_{\mathrm{H} 1}+\frac{1}{2}\left(x_{1} V_{\mathrm{H} 1}\right)^{2}+2 x_{0} V_{\mathrm{H} 0} x_{2} V_{\mathrm{H} 2}+x_{1} V_{\mathrm{H} 1} x_{2} V_{\mathrm{H} 2}\right) \\
& \left.+\frac{1}{2} E_{\mathrm{DS}} W_{\mathrm{I}} x_{1} N_{1}\right] \\
x_{2}^{\prime}=\frac{1}{m} & \frac{E_{\mathrm{B}}}{T_{\mathrm{B}}}\left(\frac{1}{4}\left(x_{1} V_{\mathrm{B} 1}\right)^{2}+x_{1} V_{\mathrm{B} 1} x_{2} V_{\mathrm{B} 2}+\left(x_{2} V_{\mathrm{B} 2}\right)^{2}\right) \\
& +\frac{E_{\mathrm{H}}}{T_{\mathrm{H}}}\left(\frac{1}{4}\left(x_{1} V_{\mathrm{H} 1}\right)^{2}+x_{1} V_{\mathrm{H} 1} x_{2} V_{\mathrm{H} 2}+\left(x_{2} V_{\mathrm{H} 2}\right)^{2}\right) \\
& \left.+E_{\mathrm{DS}} W_{\mathrm{I}}\left(\frac{1}{4} x_{1} N_{1}+x_{2} N_{2}\right)\right]
\end{aligned}
$$

where $T_{\mathrm{B}}$ is the total frequency of bee visits to all genotypes, $T_{\mathrm{H}}$ is the total frequency of hummingbird visits to all genotypes, and $m$ is the total contribution of all individuals to the next generation (mean fitness):

$$
\begin{aligned}
& T_{\mathrm{B}}=x_{0} V_{\mathrm{B} 0}+x_{1} V_{\mathrm{B} 1}+x_{2} V_{\mathrm{B} 2}, \\
& T_{\mathrm{H}}=x_{0} V_{\mathrm{H} 0}+x_{1} V_{\mathrm{H} 1}+x_{2} V_{\mathrm{H} 2}, \\
& m=E_{\mathrm{B}} T_{\mathrm{B}}+E_{\mathrm{H}} T_{\mathrm{H}}+E_{\mathrm{DS}} W_{\mathrm{I}}\left(N_{0}+N_{1}+N_{2}\right) .
\end{aligned}
$$

Equations (1)-(6) are essential to both the boundary and the branching process analyses.

\section{Boundary Analysis}

To find the conditions where the hummingbird allele $(a)$ will deterministically increase in frequency when rare, we performed a linear boundary analysis (at the initial boundary, $A A$ is fixed within the population: $x_{0} \rightarrow 1, x_{1} \rightarrow 0$, and $\left.x_{2} \rightarrow 0\right)$. Setting $x_{0}=1-x_{1}-x_{2}$, we find approximate linear equations for $\Delta x_{1}$ and $\Delta x_{2}$ in terms of $x_{1}$ and $x_{2}$, where $\Delta x_{1}=x_{1}^{\prime}-x_{1}, \Delta x_{2}=x_{2}^{\prime}-x_{2}$. We simplify these linear terms around the boundary $x_{1}=x_{2}=0$. These coefficients are vector elements in equation (7):

$$
\left[\begin{array}{l}
\Delta x_{1} \\
\Delta x_{2}
\end{array}\right]=\left[\begin{array}{ll}
L_{11} & L_{12} \\
L_{21} & L_{22}
\end{array}\right]\left[\begin{array}{l}
x_{1} \\
x_{2}
\end{array}\right],
$$


where

$$
\begin{aligned}
& L_{11}= \\
& \frac{-E_{\mathrm{B}} P(1-Z) h \alpha+E_{\mathrm{H}} P Z h \alpha+\frac{1}{2} E_{\mathrm{DS}} W_{\mathrm{I}}(-2 P Z h \alpha-P Z \alpha+P h \alpha+P-1)}{E_{\mathrm{B}} P(1-Z)+E_{\mathrm{H}} P Z(1-\alpha)+E_{\mathrm{DS}} W_{\mathrm{I}}(P Z \alpha-P+1)}, \\
& L_{12}=\frac{2\left(E_{\mathrm{B}} P(1-Z)(1-\alpha)+E_{\mathrm{H}} P Z\right)}{E_{\mathrm{B}} P(1-Z)+E_{\mathrm{H}} P Z(1-\alpha)+E_{\mathrm{DS}} W_{\mathrm{I}}(P Z \alpha-P+1)}, \\
& L_{21}=\frac{E_{\mathrm{DS}} W_{\mathrm{I}}(-2 P Z h \alpha+P Z \alpha+P h \alpha-P+1)}{4\left(E_{\mathrm{B}} P(1-Z)+E_{\mathrm{H}} P Z(1-\alpha)+E_{\mathrm{DS}} W_{\mathrm{I}}(P Z \alpha-P+1)\right.}, \\
& L_{22}=-1+\frac{E_{\mathrm{DS}} W_{\mathrm{I}}(-P Z \alpha+P \alpha-P+1)}{E_{\mathrm{B}} P(1-Z)+E_{\mathrm{H}} P Z(1-\alpha)+E_{\mathrm{DS}} W_{\mathrm{I}}(P Z \alpha-P+1)} .
\end{aligned}
$$

If the dominant eigenvalue, $\lambda$, for the matrix of equation (7) is positive, the $a$ allele can increase when rare ( $\lambda$ is a complicated quadratic equation). Figure 1 depicts the conditions for $a$ allele invasion for different values of $E_{\mathrm{Ds}}$. The most restrictive conditions for $a$ allele invasion are when plants are SI $\left(E_{\mathrm{DS}}=0\right)$. When plants are SC, the conditions for invasion are broadened, increasing according to the realized gain of delayed selfing $\left(E_{\mathrm{DS}} \cdot W_{\mathrm{I}}\right)$. The conditions for invasion do not depend on the attraction coefficient $(\alpha)$, dominance $(h)$, or the overall availability of pollinators $(P)$, although these parameters do affect the rate at which the $a$ allele increases or is lost in the population. This pattern holds if we relax the assumption of a symmetric trade-off between hummingbird versus bee attraction (app. A).

When $a$ is fully recessive $(h=0)$ and the population is $\mathrm{SI}\left(E_{\mathrm{DS}}=0\right)$, the linear approximations simplify to the fol-

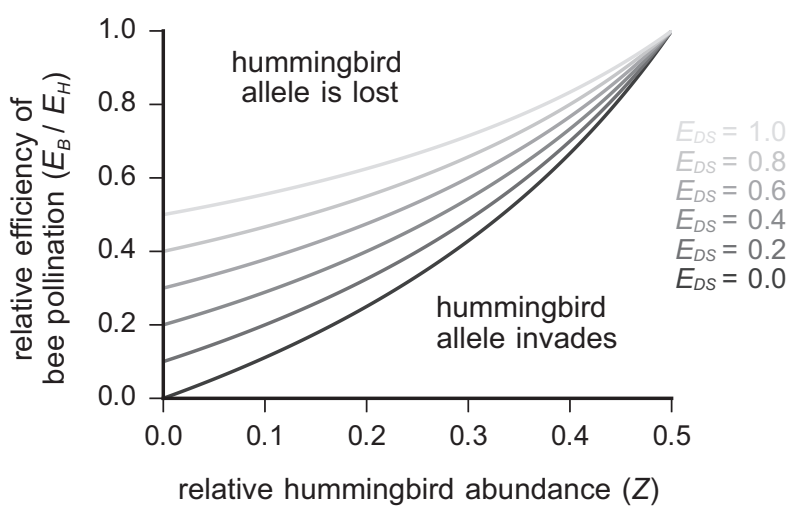

Figure 1: Conditions where the allele conferring hummingbird attraction (hummingbird allele) invades, for different values of realized efficiency of selfing $\left(E_{\mathrm{DS}}\right)$. Lines show threshold values of the relative efficiency of bee pollination below which the hummingbird allele can invade the population as a function of the proportion of pollinators that are hummingbirds $(Z)$. Here $W_{\mathrm{I}}=0.5$. lowing equation (eq. [12]), where $\lambda=0$ regardless of parameter values:

$\left[\begin{array}{l}\Delta x_{1} \\ \Delta x_{2}\end{array}\right]=\left[\begin{array}{cc}0 & \frac{2\left(E_{\mathrm{B}}(1-Z)(1-\alpha)+E_{\mathrm{H}} Z\right)}{E_{\mathrm{B}}(1-Z)+E_{\mathrm{H}} Z(1-\alpha)} \\ 0 & -1\end{array}\right]\left[\begin{array}{l}x_{1} \\ x_{2}\end{array}\right]$.

For this special case, we expand the Taylor series approximation for this boundary to consider terms that depend on $x_{1}^{2}$ (the frequency of $a$ is sufficiently small at this boundary that terms that depend on $x_{1} x_{2}$ and $x_{2}^{2}$ are negligible):

$$
\begin{aligned}
\Delta x_{1} & =\frac{2\left(E_{\mathrm{B}}(1-Z)(1-\alpha)+E_{\mathrm{H}} Z\right)}{E_{\mathrm{B}}(1-Z)+E_{\mathrm{H}} Z(1-\alpha)} x_{2}-\frac{1}{2} x_{1}^{2}, \\
\Delta x_{2} & =-x_{2}+\frac{1}{4} x_{1}^{2} .
\end{aligned}
$$

Equation (14) returns the intuitive result that, in SI populations, the production of $a a$ offspring is equal to $x_{1}^{2} / 4$ in a given generation. We can therefore substitute $x_{1}^{2} / 4$ for $x_{2}$ in equation (13) to find a simple expression for $\Delta x_{1}$ under these conditions:

$$
\Delta x_{1}=\frac{\alpha\left(-E_{\mathrm{B}}(1-Z)+E_{\mathrm{H}} Z\right)}{2\left(E_{\mathrm{B}}(1-Z)+E_{\mathrm{H}} Z(1-\alpha)\right)} x_{1}^{2} .
$$

In this case, the $a$ allele can invade when equation (15) is positive, which is the case as long as

$$
E_{\mathrm{H}} Z>E_{\mathrm{B}}(1-Z) \text {. }
$$

When we adjust our model to consider selfing that occurs before or during the opportunity for cross-pollination (prior and competing selfing), we find that these modes of selfing do not expand the conditions where the hummingbird allele can increase when rare (app. A).

\section{Branching Process}

The boundary analysis finds the conditions where the hummingbird allele will deterministically increase when rare. It does not treat the initial stochastic processes that dominate when an allele is introduced as a single copy. Evolutionary theory predicts that these stochastic processes filter the types of alleles that are most likely to contribute to adaptation (Haldane 1927) and that the fate of new recessive alleles strongly depends on mating system (Charlesworth 1992). To test our hypothesis that recessive mutations conferring hummingbird adaptation are more likely to fix when plants are capable of delayed selfing, we calculate the probability that the hummingbird allele $(a)$ can escape stochastic loss using a branching process approach. We ignore cases of overdominance; as a consequence, the probability of escape is the probability of fixation. Simplified equations (1)-(6) find $x_{1}^{\prime}$ and $x_{2}^{\prime}$ 
when $A a$ and $a a$ are present as single individuals in a population otherwise fixed for the $A$ allele:

$$
\begin{aligned}
x_{1}^{\prime}=\frac{1}{\hat{m}}[ & \left(E_{\mathrm{B}} V_{\mathrm{B} 1}+E_{\mathrm{H}} V_{\mathrm{H} 1}+\frac{1}{2} E_{\mathrm{DS}} W_{\mathrm{I}} N_{1}\right) x_{1} \\
& \left.+2\left(E_{\mathrm{B}} V_{\mathrm{B} 1}+E_{\mathrm{H}} V_{\mathrm{H} 1}\right) x_{2}\right], \\
x_{2}^{\prime}=\frac{1}{\hat{m}} & {\left[\frac{1}{4} E_{\mathrm{DS}} W_{\mathrm{I}} N_{1} x_{1}+E_{\mathrm{DS}} W_{\mathrm{I}} N_{2} x_{2}\right] . }
\end{aligned}
$$

where

$$
\hat{m}=E_{\mathrm{B}} V_{\mathrm{B} 0}+E_{\mathrm{H}} V_{\mathrm{H} 0}+E_{\mathrm{DS}} W_{\mathrm{I}} N_{0} .
$$

Assuming a Poisson distribution of offspring per parent and a stationary population size, the theory of multipoint branching processes (Feller 1968; Pollak 1987) yields equations for $U_{1}$, the fixation probability for the $a$ allele starting from a single $A a$ individual, and $U_{2}$, the fixation probability for the $a$ allele starting from a single $a a$ individual:

$$
\begin{aligned}
-\ln \left(1-U_{1}\right)= & \left(E_{\mathrm{B}} V_{\mathrm{B} 1}+E_{\mathrm{H}} V_{\mathrm{H} 1}+\frac{1}{2} E_{\mathrm{DS}} W_{\mathrm{I}} N_{1}\right) U_{1} \\
& +\frac{1}{4} E_{\mathrm{DS}} W_{\mathrm{I}} N_{1} U_{2}, \\
-\ln \left(1-U_{2}\right)= & 2\left(E_{\mathrm{B}} V_{\mathrm{B} 1}+E_{\mathrm{H}} V_{\mathrm{H} 1}\right) U_{1}+E_{\mathrm{DS}} W_{\mathrm{I}} N_{2} U_{2} .
\end{aligned}
$$

While $U_{1}$ is the relevant probability, the equations must be numerically solved simultaneously. Here we used the nsolve function of SymPy (Meurer et al. 2017), and example Python scripts used for solving equations relevant to both the boundary analysis and the branching process have been deposited in the Dryad Digital Repository: http://dx.doi.org /10.5061/dryad.8hc64 (Wessinger and Kelly 2018).

The fixation probability $\left(U_{1}\right)$ for the hummingbird allele is positive (with one specific exception; see below) within the parameter space where the $a$ allele can invade according to the deterministic boundary analysis. For any given combination of values for the proportion of hummingbirds $(Z)$, the relative efficiency of bee pollination $\left(E_{\mathrm{B}}\right)$, and the relative efficiency of hummingbird pollination $\left(E_{\mathrm{H}}\right)$ within this parameter space, the fixation probability depends positively on dominance $(h)$ and the attraction coefficient ( $\alpha$; see fig. 2 for an SI population). If plants are SI $\left(E_{\mathrm{DS}}=0\right)$, the fixation probability does not depend on pollinator availability $(P)$. In such populations, the fixation probability of a fully recessive allele is apparently zero (fig. 2), similar to a completely neutral allele (the branching process analysis breaks down at this point).

If plants are SC, pollinator availability $(P)$ and the realized gain from delayed selfing $\left(E_{\mathrm{DS}} \cdot W_{\mathrm{I}}\right)$ additionally affect the fixation probability, although their effects depend on the dominance $(h)$ of the hummingbird allele (fig. 3). The fixa-

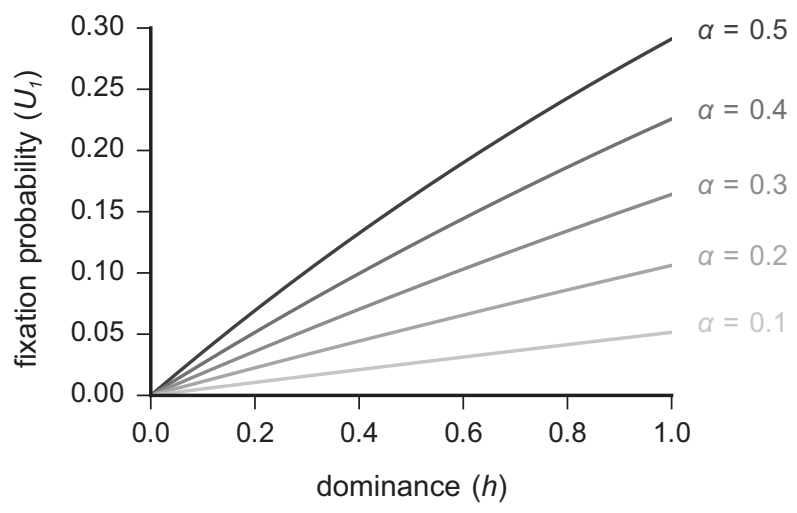

Figure 2: Fixation probability of the allele conferring hummingbird attraction starting from a single $A a$ individual $\left(U_{1}\right)$ in a self-incompatible population as a function of the dominance coefficient $(h)$, for different values of attractiveness coefficient $(\alpha)$. Here $Z=0.4, E_{\mathrm{B}}=0.4, E_{\mathrm{H}}=$ 1.0 , and $E_{\mathrm{DS}}=0$.

tion probability for dominant and partially dominant alleles is always positively related to pollinator availability $(P ;$ fig. $3 A$, $3 B)$. An increased gain from delayed selfing $\left(E_{\mathrm{DS}} \cdot W_{\mathrm{I}}\right)$ can either increase or decrease the fixation probability for such alleles, depending on the value of $P$ (in other words, depending on the selfing rate). If pollinators are more abundant than a threshold value for $P$, the gain from self-fertilization $\left(E_{\mathrm{DS}} \cdot W_{\mathrm{I}}\right)$ has a positive effect on the fixation probability, and if pollinators are less abundant than this threshold, $E_{\mathrm{DS}} \cdot W_{\mathrm{I}}$ has a negative effect on the fixation probability (fig. $3 A, 3 B$ ). The threshold value of $P$ that determines the sign of the relationship between $E_{\mathrm{DS}} \cdot W_{\mathrm{I}}$ and the fixation probability depends on the degree of dominance $(h)$ : it is greater for more dominant hummingbird alleles.

Recessive hummingbird alleles have a nonzero fixation probability if plants are SC. Unlike dominant and partially dominant alleles, the fixation probability for recessive alleles is not monotonic with respect to pollinator availability $(P)$. Instead, the maximum fixation probability occurs at intermediate $P$ (fig. $3 C$ ). The value of $P$ that maximizes the fixation probability, as well as the relationship between $P$ and the fixation probability, depends on the realized gain from self-fertilization $\left(E_{\mathrm{DS}} \cdot W_{\mathrm{I}}\right)$ : greater values of $E_{\mathrm{DS}} \cdot W_{\mathrm{I}}$ yield elevated fixation probabilities over a broad range of values of $P$ (fig. 3C). These patterns hold up when we relax the severity of the trade-off between hummingbird and bee attraction, although the fixation probabilities increase and the fixation probability of dominant and partially dominant alleles is higher for SI populations than for SC populations (app. A). When we adjust our model to consider prior and competing selfing, recessive alleles have elevated fixation probabilities in the presence of selfing, consistent with the results from delayed selfing (app. A). In this adjusted model, dominant and partially dominant alleles have a higher fixation 

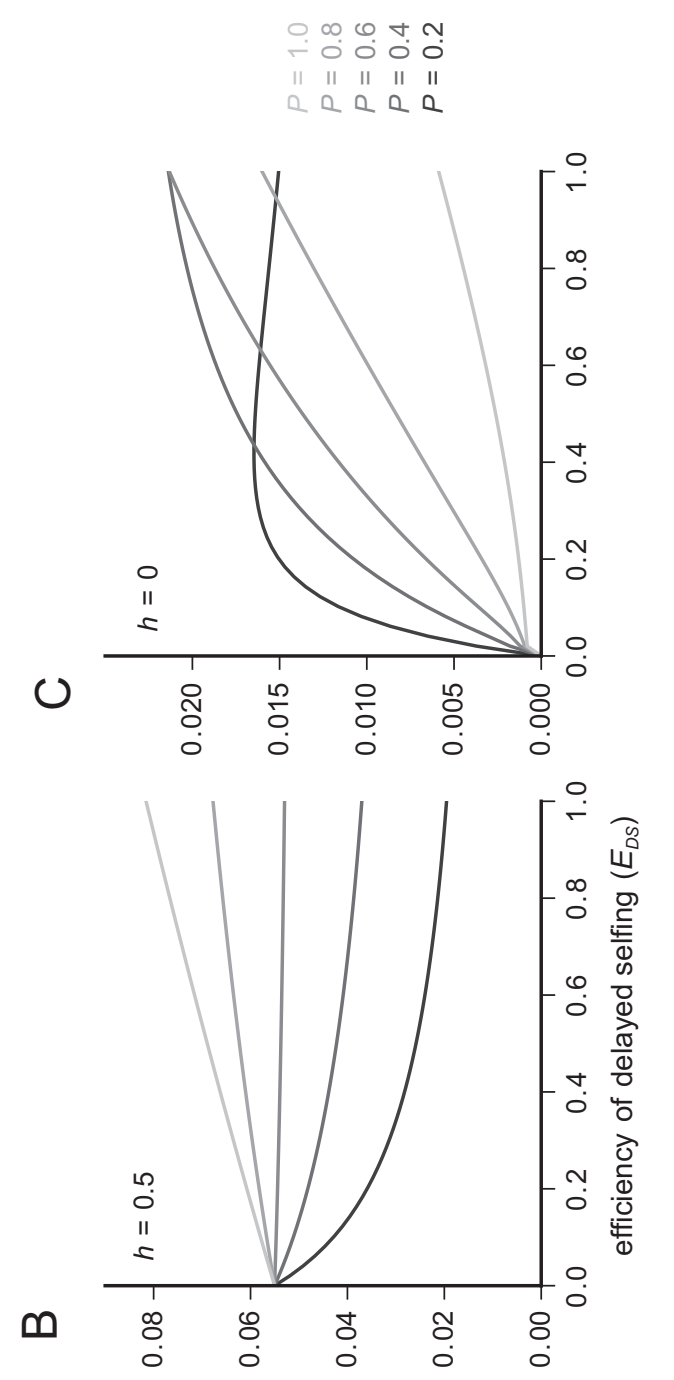

(․)

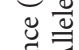

잔

ह

듕

竎 II

․․ㅇ

额

.

害弯

की

苛

语

氙

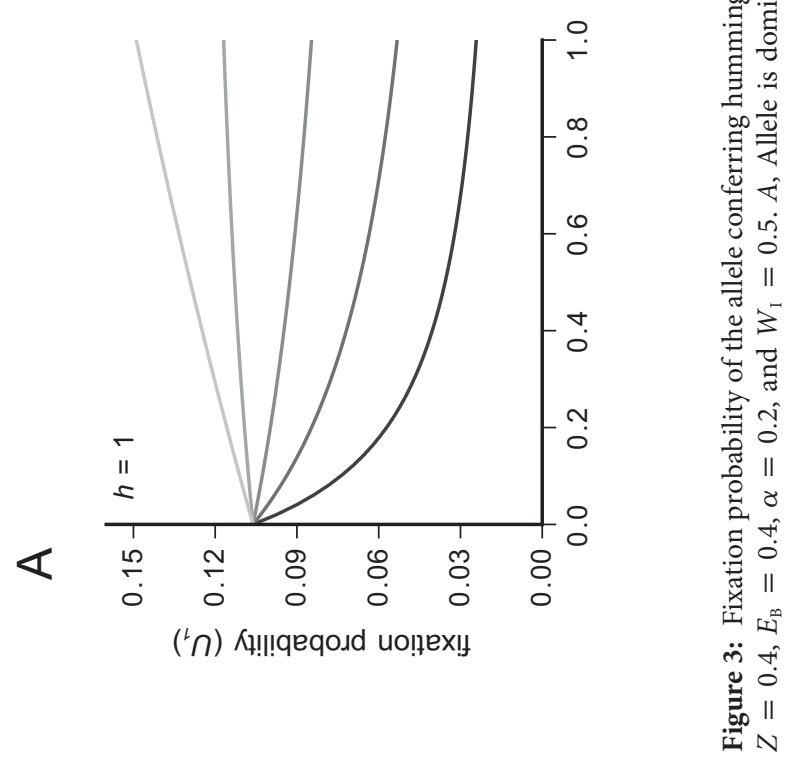


probability in the absence of selfing (app. A), consistent with the case for delayed selfing when pollinators are limited (small values of $P$ ).

\section{Evidence for an Association between Mating System and Pollinator Shifts}

We analyzed data from recent surveys of plant mating systems to assess whether an SC mating system is associated with hummingbird pollination. The index of self-incompatibility (ISI) can be defined as $1-$ (selfed seed set)/(outcross seed set), and species with ISI $<0.8$ are considered SC (Lloyd 1965; Raduski et al. 2012). In a sample of 73 Neotropical hummingbird syndrome species from 15 plant families, 54 (74\%) are SC (Wolowski et al. 2013). A sample of 1,238 angiosperms from 144 plant families collected without respect to pollination syndrome contained 695 SC species (56\%; Raduski et al. 2012). Even though the latter data set includes highly selfing species, the proportion of SC species in the hummingbird syndrome species data set is significantly higher than that in the general angiosperm data set $\left(\chi^{2}=8.24, \mathrm{df}=3, p=.002\right)$.

This comparison does not include a correction for phylogenetic relatedness. To control for the effects of phylogeny, we identified phylogenetically independent pairs of taxa that contrast bee versus hummingbird pollination and compared mating systems. We generated a phylogeny that includes all taxa in Wolowski et al. (2013) and Raduski et al. (2012) using the Open Tree of Life (Hinchliff et al. 2015). For each of the 73 hummingbird-pollinated species in the Wolowski et al. (2013) data set, we determined the most closely related species that is reported to be bee pollinated (and included in Raduski et al. 2012). This resulted in 17 clear pairs of hummingbird- versus bee-pollinated taxa (for details on our approach, see app. B). We tabulated the mating system for each of these species (app. B). In 15 of 17 cases, the hummingbird-pollinated species is SC. The bee-pollinated species is SC in only 10 of 17 cases. In six of seven cases where the mating system is different between members of a pair, the hummingbird-pollinated species is SC and the bee-pollinated species is SI.

\section{Discussion}

\section{Invasion Conditions for a Hummingbird Allele Depend on Mating System}

This article extends existing theory to consider the potentially important effect of self-fertilization on the evolutionary dynamics of pollinator attraction. Consistent with previous models of floral adaptation that have been presented within the context of floral specialization (Stebbins 1970; Waser et al. 1996; Aigner 2001), we find that an obligately outcrossing (SI) plant population will adapt to the pollinator in the community with the greatest relative abundance multiplied by the relative efficiency of pollen transfer. We find that the capacity for delayed selfing can facilitate a transition to a more efficient pollinator such as hummingbirds, even when they are less abundant in the pollinator environment, by broadening the conditions that favor an allele for hummingbird adaptation. This pattern is robust when we relax the assumption of a symmetric trade-off in pollinator attraction.

How does delayed selfing facilitate adaptation to hummingbird pollination? Adaptation to a rare pollinator carries an increased risk of not being visited by any pollinator. In SI plants, adaptation to hummingbirds, even if rare, can be favored if increased seed set from hummingbird pollination compensates for a greater risk of not being visited. Our model suggests that delayed selfing additionally compensates for this increased risk and expands the conditions that favor hummingbird adaptation. Delayed selfing confers reproductive assurance that can increase the favorability of a transition to a rare but efficient pollinator. We find that prior and competing selfing, where self-fertilization occurs before or during the opportunity to outcross, does not provide this same benefit. This suggests that delayed selfing enables the evolution of hummingbird adaptation under expanded ecological conditions not because it allows selfing per se but because it provides reproductive assurance in the absence of pollinator visitation.

\section{Effects of Delayed Selfing on the Fixation Probability of Beneficial Mutations}

For SI plants, our stochastic analyses recovered the effect of Haldane's sieve: the fixation probability of a fully recessive hummingbird allele is similar to that of a neutral allele. This result is intuitive, since a recessive allele is effectively neutral when it initially arises in an outcrossing population. Selfing immediately generates homozygotes for the hummingbird allele that can express hummingbird attraction, and in SC plants fully recessive hummingbird alleles can have a positive - and appreciable - fixation probability. This fixation probability depends on the relative contribution of delayed selfing to the next generation, a function of the opportunity for delayed selfing and its efficiency. Generally, the fixation probability for recessive hummingbird alleles is highest for populations experiencing at least moderate amounts of pollinator limitation and with relatively efficient selfpollination. In other words, as the rate and efficiency of selffertilization increases, the fixation probability for recessive hummingbird alleles is elevated. Prior or competing selfing generates effects similar to those of delayed selfing on the fixation probability of beneficial recessive alleles, suggesting that the fixation probability is a function of the effective selfing rate, regardless of the mode of selfing. This is consistent with a simpler model by Charlesworth (1992) demonstrat- 
ing that the fixation probability of a beneficial recessive allele depends on the rate of self-fertilization. These predictions are robust when we relax symmetry assumptions.

In contrast to the case for recessive alleles, the fixation probability for dominant or partially dominant hummingbird alleles is negatively affected by delayed selfing. Obligate outcrossing maximizes the number of individuals carrying the hummingbird allele, which buffers against the stochastic loss of gene copies. When pollinators are rare and delayed selfing, if expressed, is a large proportion of total reproduction, a dominant or partially dominant hummingbird allele has its highest probability of fixation with obligate outcrossing. The exception is when pollinators are sufficiently abundant so that an SC population is largely reproducing by outcrossing. In this case, delayed selfing can boost the fixation probability of a dominant or partially dominant hummingbird allele. The threshold pollinator abundance above which delayed selfing boosts the fixation probability increases with the degree of dominance. When prior or competing selfing is modeled in place of delayed selfing, the fixation probability for dominant and partially dominant alleles is always inversely related to the selfing rate, even when pollinators are abundant.

Previous work has also demonstrated that dominant beneficial alleles have a higher fixation probability in obligate outcrossing populations, whereas recessive beneficial alleles have a higher fixation probability in predominantly selfing populations (fig. 1 in Charlesworth 1992). It should be noted that, although the advantage of dominant alleles in SI populations is interesting, it is relatively minor compared with the effect of mating system on the fate of recessive alleles. Dominant and partially dominant alleles all have relatively large fixation probabilities, regardless of whether plants express delayed selfing. However, the effects of mating system on the fate of new recessive hummingbird alleles are dramatic, increasing the fixation probability from effectively zero to a nontrivial positive value. Genetic studies have found that recessive alleles that correspond to LOF mutations frequently underlie adaptations for hummingbird attraction, including red flowers, loss of floral scent, and loss of UV-absorbing pigments (table 1). Our results suggest that such recessive alleles are unlikely to fix, starting from a single copy, unless the population is capable of inbreeding. This is true regardless of the magnitude of the trade-off between hummingbird versus bee attraction.

\section{Potential Correlation between SC Mating System and the Evolution of Hummingbird Pollination}

Our model predicts that hummingbird pollination evolves more frequently in SC lineages of plants than in SI lineages, a hypothesis to be tested with comparative data. Phylogenetic tests for correlations between traits employ ancestral node reconstruction in some form, generally assuming that rates of gain and loss of a trait are equal and that traits do not strongly affect the diversification rate (e.g., Maddison 1990; Pagel 1994). However, the evolution of self-compatibility from an SI mating system is characteristically rapid and is likely unidirectional in most cases (Igić et al. 2008). In addition, the evolution of selfing affects species diversification rates (Goldberg et al. 2010). The evolution of hummingbird syndrome from bee-pollinated flowers also occurs more frequently than transitions in the reverse direction (Thomson and Wilson 2008; Barrett 2013), and an open question is whether pollination syndrome affects species diversification rates. These features complicate model-based phylogenetic tests for correlated evolution. The ideal comparative analysis would involve a large, well-resolved phylogeny of closely related species containing multiple origins of hummingbird pollination with mating system data for each tip. Such a data set does not, to our knowledge, currently exist, but we expect that numerous data sets will be established given ongoing advances in both angiosperm reproductive biology and phylogenetic systematics.

If hummingbird pollination evolves more frequently in SC lineages, we expect a relatively higher frequency of selfcompatibility in hummingbird-adapted species than in plants that rely on other animal pollinators, a prediction supported by a phylogenetically uncorrected comparison. Using a relatively small number of phylogenetically independent contrasts of related hummingbird- versus bee-pollinated species, we find that when members of a contrast differ in mating system, the hummingbird-pollinated species is SC and the beepollinated species is SI in six of seven contrasts. This clear trend is in the same direction as the larger uncorrected comparison. This suggests that the prevalence of the SC mating system in a survey of mating systems among hummingbirdpollinated plants is not simply driven by phylogenetic relatedness (Wolowski et al. 2013).

A more informal survey suggests that plant genera that have repeatedly evolved hummingbird syndrome are often predominantly SC. The wildflower genus Penstemon has evolved hummingbird syndrome flowers from the ancestral bee syndrome at least 12 independent times and perhaps as many as 20 times (Wolfe et al. 2006; Wilson et al. 2007; Wessinger et al. 2016). This remarkable pattern of repeated evolution suggests that Penstemon is prone to evolving hummingbird-adapted flowers. Mating system has been characterized in 15 Penstemon species (app. B). Each of these species is SC and can set seed when manually self-pollinated, although rates of autonomous selfing vary considerably (app. B). Hummingbird syndrome flowers have evolved seven or eight times in Costus from bee-adapted flowers (Kay et al. 2005; Salzman et al. 2015), a genus that is SC (Schemske 1983; Kay and Schemske 2008). Aquilegia and Mimulus are two predominantly SC genera that have each experienced two evolu- 
tionary transitions from bee to hummingbird syndrome (Miller 1978; Miller and Willard 1983; Eckert and Schaefer 1998; Beardsley et al. 2003; Whittall and Hodges 2007; Wu et al. 2008). At least one exception to this emerging pattern is Ipomopsis, a predominantly SI genus that has likely evolved hummingbird syndrome flowers more than once (Waser and Price 1991; LaDoux and Friar 2006; Porter et al. 2010). We suggest that any of these large genera with multiple origins of hummingbird pollination would be excellent study systems for a rigorous phylogenetic analysis using complete pollinator and mating system data.

If future comparative studies corroborate the correlation between self-compatibility and hummingbird pollination, we emphasize that this remains simply a correlation. An association between self-compatibility and hummingbird pollination could arise because self-compatibility reliably precedes or reliably follows the evolution of hummingbird pollination. This association has been discussed previously (Fenster and Martén-Rodriguez 2007; Pérez et al. 2009; Martén-Rodríguez and Fenster 2010; Martén-Rodríguez et al. 2010; Wolowski et al. 2013), with the suggested mechanism that the evolution of self-compatibility follows a pollination transition to ameliorate reproductive insecurity (Fenster and Martén-Rodriguez 2007). Our model suggests an alternative (or perhaps complementary) explanation for this association: that self-compatibility can be a preadaptation for certain types of pollinator transitions or the evolution of pollinator specialization.

\section{Conclusion}

Transitions from SI to SC mating systems are exceedingly common in plant evolution (Stebbins 1974; Igić et al. 2008), and the population genetic and macroevolutionary consequences of transitions to a predominantly selfing mating system have been a major focus of empirical and theoretical work (e.g., Stebbins 1957; Goldberg et al. 2010; Wright et al. 2013; Grossenbacher et al. 2015, 2017). However, the effects of delayed selfing on floral evolution in a predominantly outcrossing mating system have received less attention (Goodwillie et al. 2010). We suggest that the capability for delayed selfing may facilitate pollinator shifts, potentially increasing evolutionary lability in pollination syndrome and contributing to trends in floral evolution. Since pollinator shifts have historically been implicated in ecological speciation (Grant and Grant 1965; Stebbins 1970; Johnson 2006; Kay and Sargent 2009), we suggest that delayed selfing may indirectly drive patterns of plant diversification through its effect on the lability of pollination systems. Our results highlight the importance of mating system for the fate of new adaptive alleles, depending on their degree of dominance. Adaptive LOF alleles arise relatively frequently due to inherently large mutational target sizes and are often nearly or fully recessive. Delayed selfing greatly improves the chances that such re- cessive mutations can underlie the evolution of pollinator attraction, providing SC species with greater access to this mode of evolution relative to obligately outcrossing species.

\section{Acknowledgments}

We thank K. Brown, L. Hileman, B. Igić, M. Orive, and R. Unckless for conversations and comments on the manuscript as well as R. Sargent, A. Winn, and two anonymous reviewers. We thank M. Holder for assistance with the Open Tree of Life. We acknowledge funding from the National Institutes of Health (F32 GM 110988-3 to C.A.W. and R01 GM073990-02 to J.K.K.) and the National Science Foundation (DEB-1542402 to C.A.W.).

\section{Literature Cited}

Abrahamczyk, S., and S. S. Renner. 2015. The temporal build-up of hummingbird/plant mutualisms in North America and temperate South America. BMC Evolutionary Biology 15:104-116.

Aigner, P. A. 2001. Optimality modeling and fitness trade-offs: when should plants become pollinator specialists? Oikos 95:177-184.

Amrad, A., M. Moser, T. Mandel, M. de Vries, R. C. Schuurink, L. Freitas, and C. Kuhlemeier. 2016. Gain and loss of floral scent production through changes in structural genes during pollinator-mediated speciation. Current Biology 26:3303-3312.

Baker, H. G. 1955. Self-compatibility and establishment after "longdistance" dispersal. Evolution 9:347-349.

Barrett, S. C. 2002. The evolution of plant sexual diversity. Nature Reviews Genetics 3:274-284.

. 2013. The evolution of plant reproductive systems: how often are transitions irreversible? Proceedings of the Royal Society B 280:20130913.

Beardsley, P. M., A. Yen, R. G. Olmstead, and J. Willis. 2003. AFLP phylogeny of Mimulus section Erythranthe and the evolution of hummingbird pollination. Evolution 57:1397-1410.

Bradshaw, H., K. G. Otto, B. E. Frewen, J. K. McKay, and D. W. Schemske. 1998. Quantitative trait loci affecting differences in floral morphology between two species of monkeyflower (Mimulus). Genetics 149:367-382.

Bradshaw, H., and D. W. Schemske. 2003. Allele substitution at a flower colour locus produces a pollinator shift in monkeyflowers. Nature 426:176-178.

Busch, J. W., and L. F. Delph. 2012. The relative importance of reproductive assurance and automatic selection as hypotheses for the evolution of self-fertilization. Annals of Botany 109:553-562.

Byers, K. J., H. Bradshaw, and J. A. Riffell. 2014. Three floral volatiles contribute to differential pollinator attraction in monkeyflowers (Mimulus). Journal of Experimental Biology 217:614-623.

Castellanos, M. C., P. Wilson, S. J. Keller, A. D. Wolfe, and J. D. Thomson. 2006. Anther evolution: pollen presentation strategies when pollinators differ. American Naturalist 167:288-296.

Castellanos, M. C., P. Wilson, and J. D. Thomson. 2003. Pollen transfer by hummingbirds and bumblebees, and the divergence of pollination modes in Penstemon. Evolution 57:2742-2752.

Charlesworth, B. 1992. Evolutionary rates in partially self-fertilizing species. American Naturalist 140:126-148. 
Cutter, A. D., and R. Jovelin. 2015. When natural selection gives gene function the cold shoulder. BioEssays 37:1169-1173.

Eckert, C. G., K. E. Samis, and S. Dart. 2006. Reproductive assurance and the evolution of uniparental reproduction in flowering plants. Pages 183-203 in L. D. Harder and S. C. H. Barrett, eds. Ecology and evolution of flowers. Oxford University Press, Oxford.

Eckert, C. G., and A. Schaefer. 1998. Does self-pollination provide reproductive assurance in Aquilegia canadensis (Ranunculaceae)? American Journal of Botany 85:919-924.

Faegri, K., and L. Van der Pijl. 1979. The principles of pollination ecology. Pergamon, Oxford.

Feller, W. 1968. An introduction to probability theory and its applications. Vol. 1. Wiley, New York.

Fenster, C. B., W. S. Armbruster, P. Wilson, M. R. Dudash, and J. D. Thomson. 2004. Pollination syndromes and floral specialization. Annual Review of Ecology, Evolution, and Systematics 35:375-403.

Fenster, C. B., and S. Martén-Rodriguez. 2007. Reproductive assurance and the evolution of pollination specialization. International Journal of Plant Sciences 168:215-228.

Fisher, R. A. 1941. Average excess and average effect of a gene substitution. Annals of Eugenics 11:53-63.

Goldberg, E. E., J. R. Kohn, R. Lande, K. A. Robertson, S. A. Smith, and B. Igić. 2010. Species selection maintains self-incompatibility. Science 330:493-495.

Goodwillie, C., R. D. Sargent, C. G. Eckert, E. Elle, M. A. Geber, M. O. Johnston, S. Kalisz, et al. 2010. Correlated evolution of mating system and floral display traits in flowering plants and its implications for the distribution of mating system variation. New Phytologist 185:311-321.

Grant, V. 1994. Historical development of ornithophily in the western North American flora. Proceedings of the National Academy of Sciences of the USA 91:10407-10411.

Grant, V., and K. A. Grant. 1965. Flower pollination in the Phlox family. Columbia University Press, New York.

Grossenbacher, D., Y. Brandvain, J. R. Auld, M. Burd, P. O. Cheptou, J. K. Conner, A. G. Grant, et al. 2017. Self-compatibility is overrepresented on islands. New Phytologist 215:469-478.

Grossenbacher, D., R. Briscoe Runquist, E. E. Goldberg, and Y. Brandvain. 2015. Geographic range size is predicted by plant mating system. Ecology Letters 18:706-713.

Haldane, J. 1927. A mathematical theory of natural and artificial selection. V. Selection and mutation. Mathematical Proceedings of the Cambridge Philosophical Society 23:838-844.

Harder, L. D., and S. D. Johnson. 2009. Darwin's beautiful contrivances: evolutionary and functional evidence for floral adaptation. New Phytologist 183:530-545.

Harder, L. D., and W. G. Wilson. 1998. A clarification of pollen discounting and its joint effects with inbreeding depression on mating system evolution. American Naturalist 152:684-695.

Hermann, K., U. Klahre, M. Moser, H. Sheehan, T. Mandel, and C. Kuhlemeier. 2013. Tight genetic linkage of prezygotic barrier loci creates a multifunctional speciation island in Petunia. Current Biology 23:873-877.

Herrera, C. M. 1987. Components of pollinator "quality": comparative analysis of a diverse insect assemblage. Oikos 50:79-90.

Hiesey, W. M., M. A. Nobs, and O. Björkman. 1971. Experimental studies on the nature of species. V. Biosystematics, genetics and physiological ecology of the Erythranthe section of Mimulus. Carnegie Institute of Washington, Washington, DC.

Hinchliff, C. E., S. A. Smith, J. F. Allman, J. G. Burleigh, R. Chaudhary, L. M. Coghill, K. A. Crandall, et al. 2015. Synthesis of phylogeny and taxonomy into a comprehensive tree of life. Proceedings of the National Academy of Sciences of the USA 112:12764-12769.

Hoekstra, H. E., and J. A. Coyne. 2007. The locus of evolution: evo devo and the genetics of adaptation. Evolution 61:995-1016.

Igić, B., R. Lande, and J. R. Kohn. 2008. Loss of self-incompatibility and its evolutionary consequences. International Journal of Plant Sciences 169:93-104.

Johnson, S. D. 2006. Pollinator-driven speciation in plants. Pages 295 310 in L. D. Harder and S. C. H. Barrett, eds. Ecology and evolution of flowers. Oxford University Press, Oxford.

Kacser, H., and J. A. Burns. 1981. The molecular basis of dominance. Genetics 97:639-666.

Kalisz, S., D. W. Vogler, and K. M. Hanley. 2004. Context-dependent autonomous self-fertilization yields reproductive assurance and mixed mating. Nature 430:884-887.

Kay, K. M., P. A. Reeves, R. G. Olmstead, and D. W. Schemske. 2005 Rapid speciation and the evolution of hummingbird pollination in Neotropical Costus subgenus Costus (Costaceae): evidence from nrDNA ITS and ETS sequences. American Journal of Botany 92: 1899-1910.

Kay, K. M., and R. D. Sargent. 2009. The role of animal pollination in plant speciation: integrating ecology, geography, and genetics. Annual Review of Ecology, Evolution, and Systematics 40:637-656.

Kay, K. M., and D. W. Schemske. 2008. Natural selection reinforces speciation in a radiation of Neotropical rainforest plants. Evolution 62:2628-2642.

Kokubun, H., M. Nakano, T. Tsukamoto, H. Watanabe, G. Hashimoto, E. Marchesi, L. Bullrich, et al. 2006. Distribution of self-compatible and self-incompatible populations of Petunia axillaris (Solanaceae) outside Uruguay. Journal of Plant Research 119:419-430.

Kondrashov, F. A., and E. V. Koonin. 2004. A common framework for understanding the origin of genetic dominance and evolutionary fates of gene duplications. Trends in Genetics 20:287-290.

LaDoux, T., and E. A. Friar. 2006. Late-acting self-incompatibility in Ipomopsis tenuifolia (Gray) V. Grant (Polemoniaceae). International Journal of Plant Sciences 167:463-471.

Lande, R., and D. W. Schemske. 1985. The evolution of self-fertilization and inbreeding depression in plants. I. Genetic models. Evolution 39:24-40.

Lange, R. S., S. A. Scobell, and P. E. Scott. 2000. Hummingbirdsyndrome traits, breeding system, and pollinator effectiveness in two syntopic Penstemon species. International Journal of Plant Sciences 161:253-263.

Lloyd, D. G. 1965. Evolution of self-compatibility and racial differentiation in Leavenworthia (Cruciferae). Contributions from the Gray Herbarium of Harvard University 195:3-134.

- 1979. Some reproductive factors affecting the selection of self-fertilization in plants. American Naturalist 113:67-79.

- 1992. Self- and cross-fertilization in plants. II. The selection of self-fertilization. International Journal of Plant Sciences 153: $370-380$

Maddison, W. P. 1990. A method for testing the correlated evolution of two binary characters: are gains or losses concentrated on certain branches of a phylogenetic tree? Evolution 44:539-557.

Martén-Rodríguez, S., and C. B. Fenster. 2010. Pollen limitation and reproductive assurance in Antillean Gesnerieae: a specialists vs. generalist comparison. Ecology 91:155-165.

Martén-Rodríguez, S., C. B. Fenster, I. Agnarsson, L. E. Skog, and E. A. Zimmer. 2010. Evolutionary breakdown of pollination specialization in a Caribbean plant radiation. New Phytologist 188:403-417. 
Meurer, A., C. P. Smith, M. Paprocki, O. Čertík, S. B. Kirpichev, M. Rocklin, A. Kumar, et al. 2017. SymPy: symbolic computing in $\mathrm{Py}-$ thon. PeerJ Computer Science 3:e103.

Miller, R. B. 1978. The pollination ecology of Aquilegia elegantula and A. caerulea (Ranunculaceae) in Colorado. American Journal of Botany 65:406-414.

Miller, R. B., and C. L. Willard. 1983. The pollination ecology of Aquilegia micrantha (Ranunculaceae) in Colorado. Southwestern Naturalist 28:157-164.

Muchhala, N., Z. Brown, W. S. Armbruster, and M. D. Potts. 2010. Competition drives specialization in pollination systems through costs to male fitness. American Naturalist 176:732-743.

Orr, H. A. 2010. The population genetics of beneficial mutations. Philosophical Transactions of the Royal Society B 365:1195-1201.

Otto, S. P., and T. Day. 2007. A biologist's guide to mathematical modeling in ecology and evolution. Vol. 13. Princeton University Press, Princeton, NJ.

Pagel, M. 1994. Detecting correlated evolution on phylogenies: a general method for the comparative analysis of discrete characters. Proceedings of the Royal Society B 255:37-45.

Pérez, F., M. T. Arroyo, and J. J. Armesto. 2009. Evolution of autonomous selfing accompanies increased specialization in the pollination system of Schizanthus (Solanaceae). American Journal of Botany 96:1168-1176.

Phadnis, N., and J. D. Fry. 2005. Widespread correlations between dominance and homozygous effects of mutations: implications for theories of dominance. Genetics 171:385-392.

Pollak, E. 1987. On the theory of partially inbreeding finite populations. I. Partial selfing. Genetics 117:353-360.

Porter, J. M., L. A. Johnson, and D. Wilken. 2010. Phylogenetic systematics of Ipomopsis (Polemoniaceae): relationships and divergence times estimated from chloroplast and nuclear DNA sequences. Systematic Botany 35:181-200.

Raduski, A. R., E. B. Haney, and B. Igić. 2012. The expression of selfincompatibility in angiosperms is bimodal. Evolution 66:12751283.

Ronfort, J., and S. Glémin. 2013. Mating system, Haldane's sieve, and the domestication process. Evolution 67:1518-1526.

Salzman, S., H. E. Driscoll, T. Renner, T. André, S. Shen, and C. D. Specht. 2015. Spiraling into history: a molecular phylogeny and investigation of biogeographic origins and floral evolution for the genus Costus. Systematic Botany 40:104-115.

Sargent, R. D., and S. P. Otto. 2005. The role of local species abundance in the evolution of pollinator attraction in flowering plants. American Naturalist 167:67-80.

Schemske, D. W. 1983. Breeding system and habitat effects on fitness components in three Neotropical Costus (Zingiberaceae). Evolution 37:523-539.

Schemske, D. W., and C. C. Horvitz. 1984. Variation among floral visitors in pollination ability: a precondition for mutualism specialization. Science 225:519-522.

Sheehan, H., M. Moser, U. Klahre, K. Esfeld, A. Dell'Olivo, T. Mandel, S. Metzger, et al. 2016. MYB-FL controls gain and loss of floral UV absorbance, a key trait affecting pollinator preference and reproductive isolation. Nature Genetics 48:159-166.

Stebbins, G. L. 1957. Self fertilization and population variability in the higher plants. American Naturalist 91:337-354.

1970. Adaptive radiation of reproductive characteristics in angiosperms. I. Pollination mechanisms. Annual Review of Ecology and Systematics 1:307-326.
1974. Flowering plants: evolution above the species level. Belknap, Cambridge, MA.

Thomson, J. D., and P. Wilson. 2008. Explaining evolutionary shifts between bee and hummingbird pollination: convergence, divergence, and directionality. International Journal of Plant Sciences 169:23-38.

Turner, J. R. 1981. Adaptation and evolution in Heliconius: a defense of neoDarwinism. Annual Review of Ecology and Systematics 12: 99-121.

Waser, N. M., L. Chittka, M. V. Price, N. M. Williams, and J. Ollerton. 1996. Generalization in pollination systems, and why it matters. Ecology 77:1043-1060.

Waser, N. M., and M. V. Price. 1991. Reproductive costs of selfpollination in Ipomopsis aggregata (Polemoniaeae): are ovules usurped? American Journal of Botany 78:1036-1043.

Wessinger, C. A., C. C. Freeman, M. E. Mort, M. D. Rausher, and L. C. Hileman. 2016. Multiplexed shotgun genotyping resolves species relationships within the North American genus Penstemon. American Journal of Botany 103:912-922.

Wessinger, C. A., L. C. Hileman, and M. D. Rausher. 2014. Identification of major quantitative trait loci underlying floral pollination syndrome divergence in Penstemon. Philosophical Transactions of the Royal Society B 369:20130349.

Wessinger, C. A., and J. K. Kelly. 2018. Data from: Selfing can facilitate transitions between pollination syndromes. American Naturalist, Dryad Digital Repository, http://dx.doi.org/10.5061/dryad.8hc64.

Wessinger, C. A., and M. D. Rausher. 2014. Predictability and irreversibility of genetic changes associated with flower color evolution in Penstemon barbatus. Evolution 68:1058-1070.

Whittall, J. B., and S. A. Hodges. 2007. Pollinator shifts drive increasingly long nectar spurs in columbine flowers. Nature 447:706-709.

Wilson, P., A. D. Wolfe, W. S. Armbruster, and J. D. Thomson. 2007. Constrained lability in floral evolution: counting convergent origins of hummingbird pollination in Penstemon and Keckiella. New Phytologist 176:883-890.

Wolfe, A. D., C. P. Randle, S. L. Datwyler, J. J. Morawetz, N. Arguedas, and J. Diaz. 2006. Phylogeny, taxonomic affinities, and biogeography of Penstemon (Plantaginaceae) based on ITS and cpDNA sequence data. American Journal of Botany 93:1699-1713.

Wolowski, M., C. F. Saad, T.-L. Ashman, and L. Freitas. 2013. Predominance of self-compatibility in hummingbird-pollinated plants in the Neotropics. Naturwissenschaften 100:69-79.

Wright, S. I., S. Kalisz, and T. Slotte. 2013. Evolutionary consequences of self-fertilization in plants. Proceedings of the Royal Society B 280:20130133.

Wu, C., D. Lowry, A. Cooley, K. Wright, Y. Lee, and J. Willis. 2008 Mimulus is an emerging model system for the integration of ecological and genomic studies. Heredity 100:220-230.

Yuan, Y.-W., J. M. Sagawa, R. C. Young, B. J. Christensen, and H. D. Bradshaw. 2013. Genetic dissection of a major anthocyanin QTL contributing to pollinator-mediated reproductive isolation between sister species of Mimulus. Genetics 194:255-263.

\section{References Cited Only in the Online Appendixes}

Barrett, S. C. 1978. Floral biology of Eichhornia azurea (Swartz) Kunth (Pontederiaceae). Aquatic Botany 5:217-228.

Barrett, S. C., D. Wilken, and W. Cole. 2000. Heterostyly in the Lamiaceae: the case of Salvia brandegeei. Plant Systematics and Evolution 223:211-219. 
Bawa, K. S., and J. H. Beach. 1983. Self-incompatibility systems in the Rubiaceae of a tropical lowland wet forest. American Journal of Botany 70:1281-1288.

Bhaskar, V. 1992. Pollination biology and fertilization in Santalum album L. (Santalaceae). Flora 187:73-78.

Chamorro, S., R. Heleno, J. M. Olesen, C. K. McMullen, and A. Traveset. 2012. Pollination patterns and plant breeding systems in the Galápagos: a review. Annals of Botany 110:1489-1501.

Clements, R. K., J. M. Baskin, and C. C. Baskin. 1999. The comparative biology of the two closely-related species Penstemon tenuiflorus Pennell and P. hirsutus (L.) Willd. (Scrophulariaceae, section Graciles). II. Reproductive biology. Castanea 64:299-309.

Cruden, R. W., L. Hermanutz, and J. Shuttleworth. 1984. The pollination biology and breeding system of Monarda fistulosa (Labiatae) Oecologia 64:104-110.

Galetto, L. 1995. Nectary structure and nectar characteristics in some Bignoniaceae. Plant Systematics and Evolution 196:99-121.

Glenne, G. 2003. Reproductive biology, hybridization isolating mechanisms, and conservation implications of two rare subspecies of Penstemon bicolor (Brandeg.) Clokey and Keck: ssp. bicolor and ssp. roseus Clokey and Keck (Scrophulariaceae s.l.) in Clark County, Nevada. MS thesis. Utah State University, Logan.

Gottsberger, G., J. M. Camargo, and I. Silberbauer-Gottsberger. 1988. A bee pollinated tropical community: the beach dune vegetation of Ilha de São Luís, Maranhão, Brazil. Botanische Jahrbücher fur Systematik 109:469-500.

Haber, W. A., G. W. Frankie, H. Baker, I. Baker, and S. Koptur. 1981. Ants like flower nectar. Biotropica 13:211-214.

Herrera, J. 1988. Pollination relationships in southern Spanish Mediterranean shrublands. Journal of Ecology 76:274-287.

Kudoh, H., and D. F. Whigham. 1998. The effect of petal size manipulation on pollinator/seed-predator mediated female reproductive success of Hibiscus moscheutos. Oecologia 117:70-79.

Landry, C. 2012. Pollinator-mediated competition between two coflowering Neotropical mangrove species, Avicennia germinans (Avicenniaceae) and Laguncularia racemosa (Combretaceae). Annals of Botany 111:207-214

Lange, R. S., and P. E. Scott. 1999. Hummingbird and bee pollination of Penstemon pseudospectabilis. Journal of the Torrey Botanical Society 126:99-106.

Lewinsohn, J. S., and V. J. Tepedino. 2007. Breeding biology and flower visitors of the rare White River penstemon, Penstemon scariosus var. albifluvis (Scrophulariaceae). Western North American Naturalist 67:232-237.

Mabry, C. 2011. Survival, reproductive output, and transplant potential of Penstemon tubiflorus (tube penstemon). Western North American Naturalist 71:17-24.

Macior, L. W. 1974. The pollination ecology of the front range of the Colorado Rocky Mountains. Melanderia 15:1-59.
McMullen, C. K. 1994. Pollinator availability: a possible explanation of inter-island floral variation in Justicia galapagana (Acanthaceae). Noticias de Galápagos 54:22-27.

Mitchell R. 1989. Is Penstemon centranthifolius truly hummingbird pollinated? Crossosoma 15:9.

Nayak, K. G., and P. Davidar. 2010. Pollination and breeding systems of woody plant species in tropical dry evergreen forests, southern India. Flora 205:745-753.

Ramirez, N., and A. Seres. 1994. Plant reproductive biology of herbaceous monocots in a Venezuelan tropical cloud forest. Plant Systematics and Evolution 190:129-142.

Rathcke, B. 1988. Flowering phenologies in a shrub community: competition and constraints. Journal of Ecology 76:975-994.

Schlumpberger, B. O., and S. S. Renner. 2012. Molecular phylogenetics of Echinopsis (Cactaceae): polyphyly at all levels and convergent evolution of pollination modes and growth forms. American Journal of Botany 99:1335-1349.

Steiner, J., A. Zillikens, R. Kamke, E. P. Feja, and D. de Barcellos Falkenberg. 2010. Bees and melittophilous plants of secondary Atlantic forest habitats at Santa Catarina Island, southern Brazil. Oecologia Australis 14:16-39.

Tepedino, V. J., S. D. Sipes, and T. L. Griswold. 1999. The reproductive biology and effective pollinators of the endangered beardtongue Penstemon penlandii (Scrophulariaceae). Plant Systematics and Evolution 219:39-54.

Tepedino, V. J., T. R. Toler, B. A. Bradley, J. L. Hawk, and T. L. Griswold. 2007. Pollination biology of a disjunct population of the endangered sandhills endemic Penstemon haydenii S. Wats. (Scrophulariaceae) in Wyoming, USA. Plant Ecology 193:59-69.

Tripp, E. A., and P. S. Manos. 2008. Is floral specialization an evolutionary dead-end? pollination system transitions in Ruellia (Acanthaceae). Evolution 62:1712-1737.

Wang, C. N., M. Möller, and Q. C. Cronk. 2004. Population genetic structure of Titanotrichum oldhamii (Gesneriaceae), a subtropical bulbiliferous plant with mixed sexual and asexual reproduction. Annals of Botany 93:201-209.

Weiss, J., A. Nerd, and Y. Mizrahi. 1994. Flowering and pollination requirements in Cereus peruvianus cultivated in Israel. Israel Journal of Plant Sciences 42:149-158.

Wolfe, A. D., A. McMullen-Sibul, V. Tepedino, L. Kubatko, T. Necamp, and S. Fassnacht. 2014. Conservation genetics and breeding system of Penstemon debilis (Plantaginaceae), a rare beardtongue endemic to oil shale talus in western Colorado, USA. Journal of Systematics and Evolution 52:598-611.

Associate Editor: Risa D. Sargent Editor: Alice A. Winn 


\section{Correction}

After the publication of "Selfing Can Facilitate Transitions between Pollination Syndromes" by Wessinger and Kelly (American Naturalist 191:582594), the authors discovered an error in equation (6) as presented in the text. The equation lacked the variables $x_{0}, x_{1}$, and $x_{2}$. The correct equation is as follows:

$$
m=E_{\mathrm{B}} T_{\mathrm{B}}+E_{\mathrm{H}} T_{\mathrm{H}}+E_{\mathrm{DS}} W_{\mathrm{I}}\left(x_{0} N_{0}+x_{1} N_{1}+x_{2} N_{2}\right) .
$$

The correct equation was used in all analyses; thus, this error has no effect on the presented results.

CAROLYN A. WESSINGER AND JOHN K. KELLY

Department of Ecology and Evolutionary Biology, University of Kansas, Lawrence, Kansas 66045

Submitted May 7, 2019; Electronically published July 2, 2019 